\title{
Dissecting the intracellular signalling and fate of a DNA nanosensor by super-resolution and quantitative microscopy
}

Received 00th January 20xx, Accepted 00th January 20xx

DOI: $10.1039 / x 0 x \times 00000 x$

Agata Glab, ${ }^{a}$ Alessandro Bertucci, ${ }^{b}$ Fabiana Martino, ${ }^{c}$ Marcin Wojnilowicz, ${ }^{\text {a }}$ Alessia Amodio, ${ }^{\mathrm{a}, \mathrm{b}}$
Mariano Venanzi, ${ }^{\mathrm{b}}$ Francesco Ricci, ${ }^{\mathrm{b}}$ Giancarlo Forte ${ }^{\mathrm{c}}$ Frank Caruso ${ }^{* a}$ and Francesca Cavalieri *a,b

DNA nanodevices have been developed as platforms for the manipulation of gene expression, delivery of molecular payloads, and detection of various molecular targets within cells and in other complex biological settings. Despite efforts to translate DNA nanodevices from the test tube (in vitro) to living cells, their intracellular trafficking and functionality remain poorly understood. Herein, quantitative and super-resolution microscopy approaches were employed to track and visualise, with nanometric resolution, the molecular interactions between a synthetic DNA nanosensor and transcription factors in intracellular compartments. Specifically, fluorescence resonance energy transfer microscopy, fluorescence correlation spectroscopy, fluorescence lifetime imaging microscopy and multicolour single-molecule localisation microscopy were employed to probe the specific binding of the DNA nanosensor to the nuclear factor kappa-light-chain-enhancer of activated $B$ cells (NF-KB). We monitored the mobility, subcellular localisation and degradation of the DNA nanosensor inside living prostate cancer PC3 cells. Super-resolution imaging enabled the direct visualisation of the molecular interactions between the synthetic DNA nanosensors and the transcription factors molecules in cells. This study represents a significant advance in the effective detection as well as understanding of the intracellular dynamics of DNA nanosensors in a complex biological milieu.

\section{Introduction}

DNA nanotechnology has enabled the construction of complex architectures that exhibit tailorable structural and functional properties. ${ }^{1-3}$ For sensing applications, optical biosensors solely made of nucleic acids, such as DNAzymes, ${ }^{4}$ aptamers, ${ }^{5}$ DNA nanomachines, ${ }^{6}$ and DNA nanoswitches, ${ }^{7-9}$ have been developed for the detection of various molecular targets within cells and in other complex biological settings. DNA nanostructures can be used as a scaffold for assembling chromophores with pre-determined orientation, distance and association number to perform photodimerisation reactions. ${ }^{10}$ Pei et al. designed a DNA nanostructure that was able to change its shape in response to specific molecular signals such as protons, adenosine triphosphate, and mercury ions. ${ }^{11}$ Krishnan and co-workers used DNA-based nanomachines to ratiometrically map spatiotemporal changes in $\mathrm{pH}$ and calcium

\footnotetext{
a. ARC Centre of Excellence in Convergent Bio-Nano Science and Technology, and the Department of Chemical Engineering, The University of Melbourne, Parkville, Victoria 3010, Australia.

E-mail: francesca.cavalieri@unimelb.edu.au, fcaruso@unimelb.edu.au

b. Dipartimento di Scienze e Tecnologie Chimiche, Università degli Studi di Roma Tor Vergata, Via della Ricerca Scientifica 1, 00133, Rome, Italy.

International Clinical Research Center (ICRC), St Anne's University Hospital, CZ65691 Brno, Czech Republic

tElectronic Supplementary Information (ESI) available: Design of Nanoswitch ${ }_{\mathrm{NF}-\mathrm{KB}}$ and control FRET sequence, FRET efficiency calculations, Nanoswitch NF-kB $_{\text {B }}$ and control FRET sequence co-localisation studies, NF-KB knockdown, ELISA, cell viability assay, FRET and FCS experimental layout, distribution of fluorescence lifetimes. See DOI: $10.1039 / x 0 x x 00000 x$
}

ions $^{12}$ or chloride ions ${ }^{13}$ concentration in living cells. DNA probes have recently been reported for monitoring dynamic and transient molecular encounters on live cell membranes through a toehold-mediated DNA strand displacement reaction. ${ }^{14}$

Despite advances in designing DNA nanosensors for probing intracellular molecular targets, ${ }^{3}$ real-time monitoring of the actual intracellular functionality of these nanodevices remains a challenging task. The intracellular environment presents conditions very different from those found in vitro. For example, to reach a cytosolic target protein a DNA nanosensor must overcome several biological barriers. These include cellular internalisation, intracellular trafficking pathways, degradation by nucleases, and competitive binding to RNA transcripts and off-target proteins. Hence, the optical signalling provided by DNA probes in either fixed or living cells must be carefully correlated to the intracellular stability of the DNA nanodevices and the accessibility of the molecular targets must be thoroughly proved.

The intracellular and extracellular signalling of DNA nanosensors can be monitored using Förster resonance energy transfer (FRET). Although FRET methods are highly sensitive and independent of instrument efficiency and probe concentration, ${ }^{15-17}$ major interferences in the fluorescence readout arise from the intracellular degradation of the DNA probe, mediated by nucleases, and from the different photophysical behaviour of the donor and acceptor in the complex intracellular milieu. In fact, these effects can result in false-positive signals. ${ }^{18-23}$ Recently, Lacroix et al. ${ }^{24}$ thoroughly 
investigated the cellular uptake and fate of fluorescently labelled DNA nanostructures. This study revealed that most of the intracellular fluorescence signal arises from the degradation of the DNA nanostructures mediated by extracellular nucleases, releasing the fluorescent dye that is then taken up by cells. These important findings suggest that when DNA nanostructures are used in cells, stringent validation methods are required. In addition, multiple and complementary imaging technologies are vital for the interpretation of the experimental results. ${ }^{24}$ For instance, quantitative and super-resolution microscopy techniques, such as fluorescence correlation spectroscopy (FCS), fluorescence lifetime imaging microscopy (FLIM), and single-molecule localisation microscopy (SMLM) are powerful means for dissecting the intracellular functionality and fate of DNA nanosensors and nanomachines, with spatial and temporal resolution.

We recently demonstrated that a DNA nanoswitch, originally designed with a fluorophore/quencher pair for the quantitative detection of transcription factors (TFs) in test tube (in vitro), ${ }^{9}$ enabled qualitative detection of nuclear factor kappa-light-chain-enhancer of activated B cells (NF-kB) in prostate cancer PC3 cells. ${ }^{7}$ The DNA nanoswitch was successfully used for real-time qualitative analysis of the siRNAregulated knockdown of NF-KB. ${ }^{7}$ Probing the expression and dynamics of eukaryotic NF-kB family of proteins, including $\mathrm{p} 65$ and $\mathrm{p50}$, in live cells is important because these TFs regulate diverse biological processes such as cell growth, immune and inflammatory responses, and apoptosis. ${ }^{25-27}$ However, quantitative intracellular analysis of the DNA nanoswitch binding activity, diffusion and degradation in living cells remained elusive. In the present study, we sought to elucidate the intracellular signalling and fate of the DNA nanoswitch, by combining different microscopy techniques including FRET imaging microscopy, FCS, FLIM and stochastic optical reconstruction microscopy (STORM). A modified DNA nanoswitch, incorporating a FRET donor-acceptor pair, was employed for probing the specific recognition of p50/p50 and p50/p65 NF-KB dimers in cells (Figure 1). Compared with the previous single-probe nanoswitch ${ }^{7}$ the modified FRET-based system exhibited higher affinity for the target proteins and enabled the visualisation of NF-KB/DNA nanoswitch complexes with nanometric resolution in cells. By using FCS and FLIM, we were able to discriminate in live cells between three species, i.e., unbound, bound, and degraded DNA nanoswitch. The present approach can be extended to other DNA nanostructures and is thus expected to advance the understanding of intracellular dynamic processes and functionality of a variety of DNA nanosensors and nanomachines.

\section{Experimental}

\section{Materials}

HPLC-purified oligonucleotide DNA sequences of Nanoswitch NF- $_{\text {- }}$ кв, 5'-[Quasar 670]-AGTATGGGACTTTCCATACTT[Quasar 570]ATTTGAGGAAAGTCCCTCAAAT-3' (the binding domain is underlined), and control FRET sequence, 5'-[Quasar 670]-

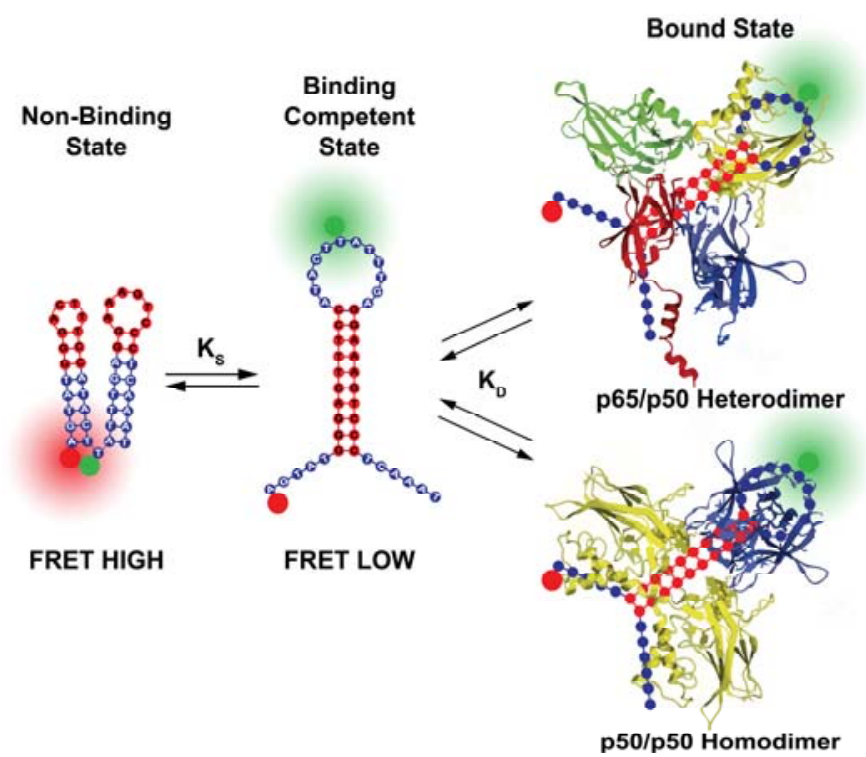

Figure 1. Schematic showing the binding of the Nanoswitch NF-kB $_{\text {to }}$ to the heterodimer $\mathrm{p} 50 / \mathrm{p} 65$ and homodimer $\mathrm{p} 50 / \mathrm{p} 50$. In the non-binding conformation on the left, the NF$\mathrm{KB}$ binding motif is unexposed and the donor-acceptor pair in close proximity ("FRET $\mathrm{HIGH}^{\prime \prime}$ ). In the conformation on the right, the binding-competent state, the binding domain of p50/p50 or p50/p65 is exposed and the donor-acceptor pair is set at a larger distance ("FRET LOW"). $K_{\mathrm{S}}$, switching equilibrium constant; $K_{\mathrm{D}}$, dissociation constant. The large red circles represent Quasar670 and green circles represent Quasar570.

AGTATTGTTTTTACAATACTT[Quasar570]ATTTGATGTTTTTTCATC AAAT-3', were supplied by Biosearch Technologies (Novato, CA, USA). Human recombinant NF-kB p50 and p65 were purchased from Cayman Chemical (Ann Arbor, MI, USA) and Aviva Systems Biology (San Diego, CA, USA), respectively. Lipofectamine ${ }^{\circledR}$ RNAiMAX transfection reagent, DNase I, DNase I buffer, 100x Halt $^{\mathrm{TM}}$ protease inhibitor cocktail, and Alexa Fluor 488, 555 and 647 dyes were obtained from Life Thermo Fischer Scientific (Waltham, MA, USA). PC3 human prostate epithelial cancer cell (CRL-1435) were purchased from ATCC ${ }^{\circledR}$. NF-KB p65 ELISA ${ }^{\mathrm{TM}}$ kit was supplied by Abcam (Cambridge, UK). Dulbecco's phosphate-buffered saline (D-PBS), 3-(4,5-dimethyl-2thiazolyl)-2,5-diphenyl-2H-tetrazolium bromide (MTT), bovine serum albumin (BSA), RIPA buffer, poly(ethylene glycol) $\left(M_{\mathrm{W}}\right.$ 3350), and anti-rabbit IgG (whole molecule)-peroxidase antibody produced in goat were supplied by Sigma-Aldrich (St. Louis, MO, USA). Opti-MEM reduced serum medium and rabbit anti-NF-kB antibody were obtained from Life Technologies (Scoresby, Australia). Rabbit anti- $\beta$-actin antibody, EEA1, Rab7, GM130 and Tom20 antibodies were purchased from Cell Signaling Technology (Danvers, USA). Goat anti-rabbit IgG secondary antibody-Alexa Fluor 488 conjugate was supplied by Invitrogen (Carlsbad, USA). Trypsin and Dulbecco's Modified Eagle's medium (DMEM) were purchased from Lonza (Allendale, USA). Fetal bovine serum (FBS) was supplied by Bovogen (Keilor East, Australia). Tween-20 was purchased from Chem-Supply (St. Gillman, Australia). siRNA targeting NF-KB (sense: 5'-GGGUAUAGCUUCCCACACU[dT][dT]-3'; anti-sense: 5'-AGUGUGGGAAGCUAUACCC[dT][dT]-3') was purchased from Sigma-Aldrich (St. Louis, MO, USA). All chemicals were used without further purification. 


\section{Binding and energy transfer properties of Nanoswitch $\mathrm{NF}_{\mathrm{NFB}}$}

Fluorescence measurements of the Nanoswitch NF-kB $_{\text {were }}$ performed on a Horiba FL-322 Fluorolog-3 spectrometer equipped with a $450 \mathrm{~W}$ xenon lamp as excitation source. All measurements were performed at $37^{\circ} \mathrm{C}$ in quartz cuvettes with a volume of $200 \mu \mathrm{L}$. The excitation wavelength, $\lambda_{\mathrm{ex}}$, was $520 \mathrm{~nm}$ (slit $=5 \mathrm{~nm}$ ), and emission was acquired in the range from 550 to $720 \mathrm{~nm}$ (slit $=5 \mathrm{~nm}$ ). Fluorescence emission spectra were obtained by incubation of $5 \mathrm{nM}$ Nanoswitch NF-kB $_{\text {or }} 5 \mathrm{nM}$ control FRET sequence with increasing concentrations of NF-KB p50/p50 homodimer or p50/p65 heterodimer. For measurements performed in PBS, the concentration varied from 0 to $250 \mathrm{nM}$, whereas for measurements performed in PEG/PBS $(30 \% \mathrm{w} / \mathrm{w})$, the concentration varied from 0 to $10 \mathrm{nM}$. Maximal fluorescence signal, originating from donor, Quasar 570 , was measured after incubation of $5 \mathrm{nM}$ Nanoswitch NF-KB $_{\text {or }}$ control FRET sequence with DNase I for $60 \mathrm{~min}$ at $37^{\circ} \mathrm{C}$.

\section{Intracellular FRET measurements}

Human prostate cancer PC3 cells were plated on Nunc ${ }^{\mathrm{TM}}$ LabTek $^{\mathrm{TM}}$ II chambered coverglass (Life Technologies, Scoresby, Australia) at a seeding density of $4 \times 10^{4}$ cells per well in $500 \mu \mathrm{L}$ of DMEM medium supplemented with $10 \%$ FBS and incubated at $37{ }^{\circ} \mathrm{C}$ in $5 \% \mathrm{CO}_{2}$ overnight. Then, transfection with Nanoswitch $h_{\text {NF-kB }}$ and the control FRET sequence was performed for $2 \mathrm{~h}$ in OPTI-MEM reduced serum medium using Lipofectamine RNAiMAX as a carrier according to supplier protocol, with the final DNA concentration varying between 5 $\mathrm{nM}$ and $100 \mathrm{nM}$. The transfection efficiency after $2 \mathrm{~h}$ incubation with the Nanoswitch ${ }_{\mathrm{NF}-\mathrm{KB}}$-Lipofectamine was found to be $25 \pm$ $4 \%$ by measuring the fluorescence signal of the supernatant medium before and after transfection. This result indicates that an incubation time of $2 \mathrm{~h}$ is a suitable period to obtain a sufficient amount of internalised Nanoswitch NF-KB $_{\text {and a reliable }}$ signal intensity in the range of concentrations examined. Flow cytometry data (Figure S1) also confirmed that after $2 \mathrm{~h}$ of transfection, $60 \pm 10 \%$ of cells had internalised the

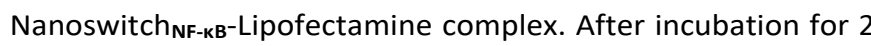

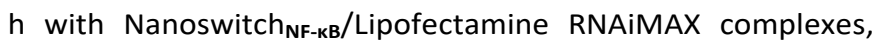
the transfection medium was discarded, and cells were washed with sterile D-PBS and incubated in fresh culture medium to keep the intracellular concentration of transfection complex constant after initial uptake. Cells were incubated for another $0,5,8$ and $22 \mathrm{~h}$ after removal of transfection medium, corresponding to time points of 2, 7, 10 and $24 \mathrm{~h}$, washed three times with D-PBS, fixed for 10 min with $4 \%$ paraformaldehyde at $25{ }^{\circ} \mathrm{C}$, washed three times with D-PBS, and resuspended in $350 \mu \mathrm{L}$ D-PBS. FRET measurements and cells imaging were performed using Nikon A1R confocal microscope with a $60 \times$ 1.4NA oil immersion objective. High voltage (gain), offset, and laser power were kept constant during data acquisition of all samples and controls. Image processing was performed using NIS-Element confocal software (Nikon). For the intracellular FRET measurements, for each condition, at least 80 cells were analysed.
Determination of a nuclear signal in the transfected cells was performed using ImageJ. To identify all cells exhibiting a nuclear signal, the image brightness was increased to the maximum so that weak nuclear fluorescence could be spotted. Then, all cells with a nuclear fluorescent signal were identified and calculated.

\section{STORM analysis}

Dual-labelled secondary antibodies were prepared as follows: $50 \mu \mathrm{L}$ secondary antibody Alexa Fluor 488 ( $2 \mathrm{mg} / \mathrm{mL}$ in PBS) was mixed with $50 \mu \mathrm{L}$ of $0.1 \mathrm{M} \mathrm{NaHCO}_{3}$ and $10 \mu \mathrm{L}$ Alexa Fluor 647 (activator dye/reporter dye $=2: 1$ ). The reaction proceeded for $30 \mathrm{~min}$ at $25^{\circ} \mathrm{C}$ in the dark while stirring on a shaking platform. The reaction volume was adjusted to $0.5 \mathrm{~mL}$. Then, the antibody was purified using NAP-5 gel filtration column equilibrated in PBS. PC3 cells, approximately 30000 , were seeded on 8 -well Lab-Tek chamber slides, followed by addition of $0.5 \mathrm{~mL}$ culture medium and incubation overnight. Transfection with Nanoswitch $_{\text {NF-KB }}$ was achieved using Lipofectamine RNAiMAX as per the transfection protocol provided by the supplier so that the final concentration of the Nanoswitch $\mathrm{NF}_{\mathrm{N}-\mathrm{KB}}$ in each well was $50 \mathrm{nM}(0.5 \mathrm{~mL}$ culture medium, 30\% DNA-lipoplex solution). After incubation for $2 \mathrm{~h}$ at $37{ }^{\circ} \mathrm{C}$ in $5 \% \mathrm{CO}_{2}$, the transfection medium was discarded, the cells were fixed and treated as previously described. Immunostaining for NF-KB was performed by incubating cells with rabbit anti-p50 primary antibody $(2.5$ $\left.\mu \mathrm{g} / \mathrm{mL}, 1 \mathrm{~h}, 25^{\circ} \mathrm{C}\right)$, washing with $1 \%$ BSA/PBS $(3 \times)$, then incubating with Alexa Fluor 647/Alexa Fluor 488 dual-labelled goat anti-rabbit secondary antibody $\left(2 \mathrm{\mu g} / \mathrm{mL}, 1 \mathrm{~h}, 25^{\circ} \mathrm{C}\right)$, and washing again with PBS (3x). STORM images were acquired using a Nikon N-STORM system configured for total internal reflection fluorescence imaging (TIRF). The perfect focus system and TIRF angle were adjusted and tuned to maximise the signalto-noise ratio. The dual labelled (Alexa Fluor 488- and Alexa Fluor 647-labelled) antibody and Nanoswitch NF-KB $_{\text {(Quasar570 }}$ Quasar670) were excited by 488,561 and $647 \mathrm{~nm}$ laser lines. No ultraviolet light activation was used. As the reporter dyes on the secondary antibody (AF647) and Nanoswitch ${ }_{\text {NF-kB }}$ (Quasar670) exhibit similar photophysical properties and quantum yields, (0.33) we assume a similar detection efficiency for both molecules. Fluorescence was collected using a Nikon 100x 1.4NA oil immersion objective and passed through a quad-band pass dichroic filter. All time lapses were recorded onto a 256 pixel $\times 256$ pixels region using an EMCCD camera. For each channel, 3000-5000 frames were sequentially acquired. STORM frames were analysed with the STORM module of the NIS Elements Nikon software. The software generates a list of localizations by Gaussian fitting the fluorescence spots of blinking dyes, allowing correction for possible mechanical drift. To rule out overcounting, blinking events detected in consecutive frames are counted as singlemolecule by the software. The number of blinking molecules on a single nanoobject in the acquired plane was obtained using the Nikon analysis software. To evaluate the number of protein molecules in the cluster, at least 100 protein aggregates were identified. To evaluate the colocalization between NF-KB and 
Nanoswitch $_{\text {NF-kB }}$ for each experimental condition, approximately 1500 protein and Nanoswitch NF-KB $_{\text {K }}$ molecules/cluster were identified; the objects were considered colocalized when their centroid positions were no more than $150 \mathrm{~nm}$ from each other. ${ }^{28}$

\section{FLIM and FCS measurements in live cells}

Human prostate cancer PC3 cells were plated on Nunc ${ }^{\mathrm{TM}}$ LabTek $^{\mathrm{TM}}$ II chambered coverglass (Life Technologies, Scoresby, Australia) at a seeding density of $4 \times 10^{4}$ cells per well and the transfection was carried out according to the protocol described before. FLIM imaging and FCS measurements were performed in 10\% FBS/Leibovitz's L-15 medium, which supports cell growth in an environment without $\mathrm{CO}_{2}$ equilibration, at 37 ${ }^{\circ} \mathrm{C}$. Fluorescence lifetime images were acquired on a Nikon A1R confocal microscope equipped with a picosecond pulsed diode laser (PDL 800-D; excitation wavelength of $647 \mathrm{~nm}$ ) and a PMA hybrid detection unit (PicoQuant $\mathrm{GmbH}$, Berlin, Germany). The decay curves were acquired and analysed using SymPhoTime software. For each condition 20 measurements were performed. FCS measurements were performed using a MicroTime PicoQuant system combined with a Nikon A1R confocal microscope equipped with either a 40× 1.1NA water immersion objective (measurements in solution) or a $60 \times 1.4 \mathrm{NA}$ oil immersion objective (measurements in cells). The Nanoswitch $_{\text {NF-KB }}$ was excited using a $561 \mathrm{~nm}$ laser and signals were detected using a 600/50 bandpass filter. The optical system was calibrated using Alexa Fluor 555 as a reference, and the diffusion coefficient $(D)$ was calculated using that of Alexa Fluor 555 as a standard $\left(300 \mu \mathrm{m}^{2} / \mathrm{s}\right)$. Fluorescence was recorded using the PMA hybrid detection unit, which was coupled to a PicoHarp 300 TCSPC module (PicoQuant GmbH, Berlin, Germany). For solution measurements, fifteen sets of data acquisition, with each set lasting for $60 \mathrm{~s}$, were recorded. In the cells, for each concentration, a minimum of 10-point measurements, with each set lasting for $20 \mathrm{~s}$, were recorded. FCS measurements were acquired and analysed using the SymPhoTime software.

\section{Results and Discussion}

\section{Binding properties of the Nanoswitch ${ }_{\mathrm{NF}-\mathrm{kB}}$ in test tube}

An optical DNA nanoswitch containing the binding sequence 27 , ${ }^{29}$ for the homodimer p50/p50 and the heterodimer p50/p65 (5'-GGGACTTTCC-3', depicted in red in Figure 1) and a donoracceptor FRET pair (Quasar 570-Quasar 670), hereafter

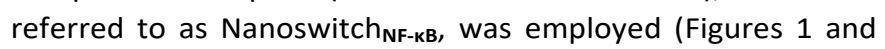
S2). We performed BLAST analysis, as described in the Supporting Information (ESI) (Section S1, Figures S3 and S4, Table S1), to exclude possible off-target interactions of the

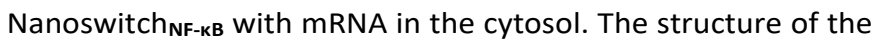

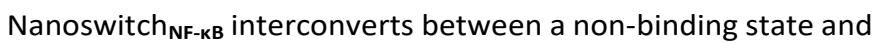
a binding-competent state (Figure 1). ${ }^{7,} 9$ The equilibrium between the two states is shifted towards the binding state upon interaction with NF-KB. The non-binding state presents the NF-KB binding motif unexposed and the FRET pair in close proximity, thus enabling efficient quenching of the donor by both contact and FRET effects (FRET HIGH). ${ }^{30}$ The bindingcompetent state exposes the NF-KB binding domain and sets the acceptor-donor pair at a distance of approximately $6 \mathrm{~nm}$ with reduced FRET effects (FRET LOW). Hence, by monitoring the experimental changes in FRET signal, the binding properties of Nanoswitch $_{\text {NF-KB }}$ can be studied. It is worth noting that the intramolecular interactions between the selected donor and acceptor FRET pair contribute to the conformational and binding equilibria of the Nanoswitch ${ }_{\mathrm{NF}-\mathrm{kB}}$.

The binding properties of the Nanoswitch NF-KB $_{\text {were }}$ first characterised in test tube. A comparative study of the binding between the Nanoswitch NF-KB $_{\text {and the }}$ a0/p50 homodimer or the $\mathrm{p50} / \mathrm{p} 65$ heterodimer was conducted in phosphate buffer saline (PBS) or poly (ethylene glycol) (PEG)/PBS $(30 \% \mathrm{w} / \mathrm{w})$ as shown in Figures $2 A$ and $S 5 A$. The binding curves and the relative dissociation constants, $K_{\mathrm{D}}$, were calculated through titration experiments and changes in the fluorescence emission of the donor and acceptor (see SI, Section S2). Upon recognition of p50/p50 or p50/p65, the fluorescence emission intensity of the donor at $570 \mathrm{~nm}$ gradually increased, whereas that of the acceptor at $658 \mathrm{~nm}$ decreased (Figure S6). Consequently, a
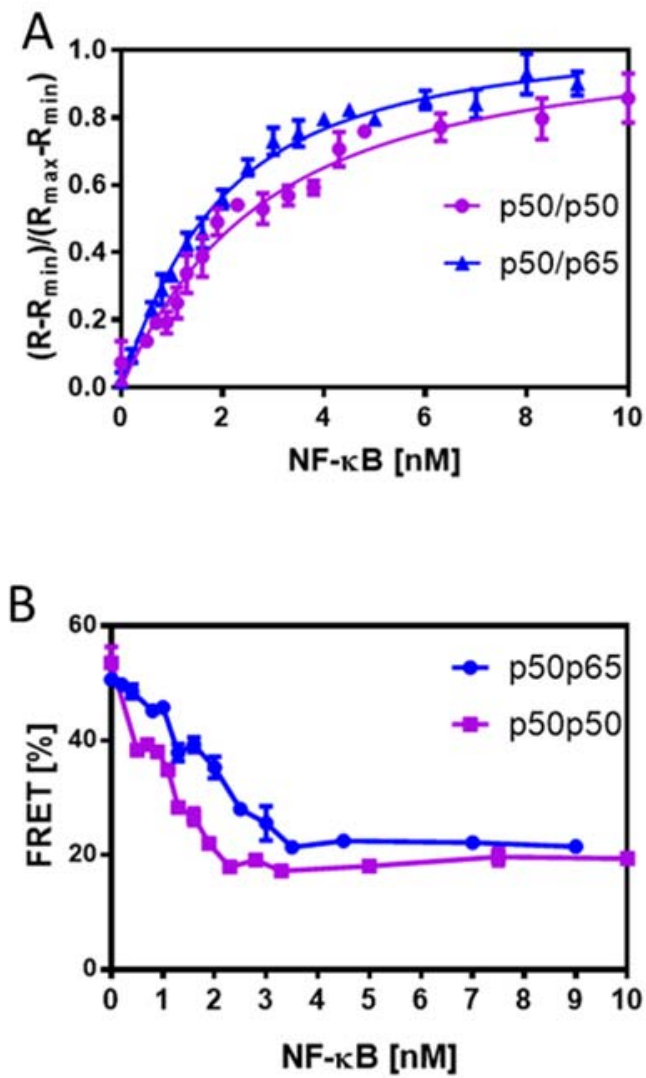

Figure 2. FRET studies performed in test tube to characterise the binding properties of the Nanoswitch ${ }_{\mathrm{NF}-\mathrm{kB}}$ to $\mathrm{p50/p50}$ and p50/p65. (A) Binding curves of the Nanoswitch $\mathrm{NF}_{\mathrm{NB}}$ $(5 \mathrm{nM})$ for the target proteins in PEG/PBS $(30 \% \mathrm{w} / \mathrm{w})$. (B) Variation in the FRET efficiency of the Nanoswitch NF-kB $(5 \mathrm{nM})$ as a function of p50/p65 and p50/p50 concentration. All the measurements were performed at $37^{\circ} \mathrm{C}$. Error bars represent standard deviations, $\mathrm{n}=3$. 
variation in the donor-to-acceptor fluorescence peak ratio $(R)$ ranging from minimum to maximum ratio values $\left(R_{\min }, R_{\max }\right)$ was observed. A stable fluorescence emission signal was achieved in less than $5 \mathrm{~min}$, indicating a fast binding kinetics. The binding curves were obtained by plotting the normalised ratiometric value $\left(R-R_{\min }\right) /\left(R_{\max }-R_{\min }\right)^{31}$ against the concentration of p50/p50 or p50/p65 dimer (Figures 2A and S5A).

To mimic the intracellular environment, $K_{\mathrm{D}}$ values were determined at $37^{\circ} \mathrm{C}$ in PEG/PBS (Figure 2A) or PBS (Figure S5A). The intracellular environment is a crowded milieu, with biological macromolecules, such as proteins, nucleic acids, and polysaccharides, occupying up to $20-40 \%$ of the cellular volume. $^{32,33}$ It is well known that crowding conditions can shift the equilibrium of association-dissociation reactions toward the associated state because of excluded volume effects and an increase in the total entropy of the system. ${ }^{32,33}$ Though exact reproduction of the complex intracellular biological conditions is not possible in test tube, macromolecular crowding is typically mimicked by concentrated solutions of PEG or other hydrophilic polymers that serve as model "crowding agents". ${ }^{33}$ As only a particular Nanoswitch ${ }_{\mathrm{NF}-\mathrm{KB}}$ conformer can bind to either $\mathrm{p} 50 / \mathrm{p} 50$ or p50/p65 (Figure 1), the titration data were analysed according to a Langmuir-type one-site binding process combined with a conformational equilibrium (see S1, Section S2, Equations (S3) and (S5)). The results indicate that the Nanoswitch $_{\text {NF-kB }}$ binds to p50/p50 and p50/p65 in PBS, with $K_{\mathrm{D}}$ values of $20 \pm 2$ and $36 \pm 4 \mathrm{nM}$, respectively. Interestingly, under the simulated crowded conditions, $K_{\mathrm{D}}$ decreased approximately one order of magnitude i.e., to $2.1 \pm 0.3$ and $1.3 \pm 0.1 \mathrm{nM}$, respectively. These data suggest that the Nanoswitch NF-KB $_{\text {binds }}$ with high and similar affinity to both p50/p50 and p50/p65 in a crowded environment.

The absolute FRET efficiencies of the Nanoswitch NF-kB $_{\text {upon }}$ binding in PBS and PEG/PBS solutions were also evaluated according to Equation (S1), (see SI, Section S2). The FRET efficiency measured in either PEG/PBS (Figure 2B) or PBS (Figure S5B) decreased from approximately $55 \%$ to $20 \%$ with increasing concentration of p50/p50 or p50/p65 dimer. These results confirm that the binding event induces a shift in equilibrium toward the FRET LOW bound state, where the donor and acceptor are set at a distance. To experimentally evaluate this distance, we first determined the FRET characteristic distance $R_{0}$ (53.4 $\AA$ ), i.e. the distance at which the donor-acceptor pair shows a $50 \%$ FRET efficiency (FRET HIGH state), then by applying the Förster Equation (see SI, Section S2, Equation S6) we estimated a distance of $67.3 \AA$ between the donor and acceptor pair, in the FRET LOW state.

As expected, the treatment of the Nanoswitch NF-kB $_{\text {with }}$ DNAse in test tube (Figure S7) resulted in a rapid abrogation of FRET effects. A "control FRET sequence" (see SI, Section S1) with double stem-loop structural features analogous to those of the Nanoswitch $_{\text {NF-KB }}$ in the FRET HIGH state, but lacking the NF-KB binding domain, was used as a negative control. As this control sequence resides in a stable conformational state, it provides a control for nonspecific binding of the Nanoswitch ${ }_{\mathrm{NF}-\mathrm{KB}}$ to $\mathrm{p} 50 / \mathrm{p} 50$ or $\mathrm{p} 50 / \mathrm{p} 65$ driven by electrostatic interactions. Only very slight variations in the control FRET efficiency (within the
80-90\% range) were observed with increasing protein concentrations up to $120 \mathrm{nM}$ (see SI, Figure S8), indicating a limited binding and absence of conformational changes. Overall, our results indicate that the Nanoswitch NF-kB $_{\text {can }}$ bind to intracellular p50/p50 or p50/p65 dimers with high affinity and the extent of the binding can be monitored by measuring absolute FRET efficiencies.

\section{Monitoring of the intracellular binding efficiency of Nanoswitch ${ }_{\mathrm{NF}-}$ ${ }_{\mathrm{B}}$ by quantitative FRET microscopy}

The target TFs, p50/p65 and p50/p50, are generally kept in an inactive latent state in the cytoplasm of most unstimulated cells. ${ }^{25}$ Conversely, cytoplasmic NF-KB is upregulated and constitutively activated in many cancer cells including prostate cancer PC3 cell. NF-KB is activated through an enzymatic cascade event that frees NF-KB from its inhibited complex to translocate into the nucleus. ${ }^{25}$ Several studies have shown that the eukaryotic TFs interact with chromatin in a dynamic fashion and are freely diffusing biomolecular species. ${ }^{34,35}$ The intracellular binding of the Nanoswitch NF-KB $_{\text {B }}$ to NF-KB was initially investigated in PC3 prostate cancer cells by confocal microscopy.

PC3 prostate cancer cells were treated with the Nanoswitch NF- $_{\text {- }}$ кв and control FRET sequence for $2 \mathrm{~h}$ using the commercial cationic lipids Lipofectamine ${ }^{\circledR}$ as transfection agent. After incubation for $2 \mathrm{~h}$, the transfection medium was discarded, the cells were grown in fresh culture medium and analysed at different time points (2-24 h).

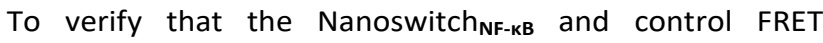
sequence transfected by lipofectamine escape from the endolysosomal vesicles and diffuse in the cytoplasm, we performed colocalisation studies with early and late endosomes/lysosomes by immunostaining of fixed cells. Confocal microscopy images (Figures S9 and S10) and calculated Pearson's correlation coefficients (PCC) showed limited colocalisation of

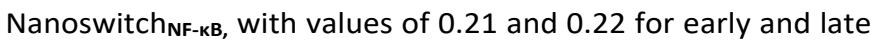
endosomes after $2 \mathrm{~h}$ incubation, and 0.13 and 0.21 for early and late endosomes after $24 \mathrm{~h}$ incubation. Similar results were obtained for cells incubated with control FRET sequence, with values of 0.16 and 0.35 for early and late endosome after $2 \mathrm{~h}$ and 0.12 and 0.15 for early and late endosome after $24 \mathrm{~h}$ incubation. In addition, we observed a negligible colocalisation of Nanoswitch ${ }_{\text {NF-kB }}$ signal with mitochondria $(P C C=0.22)$ and Golgi apparatus (PCC $=0.24$ ) after $24 \mathrm{~h}$ incubation (Figures S9 and S10). The images also show highly fluorescent spots after $24 \mathrm{~h}$ incubation, which we ascribed to the LipofectamineNanoswitch $_{\text {NF-KB }}$ complex, either internalised by the cell or attached to the cell surface. Overall, these results clearly suggest that the endosomal escape and release of Nanoswitch $_{\text {NF-kB }}$ or control FRET sequence in the cytosol occur within a few hours of incubation.

Next, the FRET signal arising from the internalised Nanoswitch $_{\text {NF-kB }}$ was monitored as a function of time. PC3 cells were transfected with different amounts of Nanoswitch NF-kB $_{\text {(5- }}$ $50 \mathrm{nM}$ ) to evaluate the concentration dependence of FRET signal. The intracellular FRET efficiencies were obtained using the acceptor photobleaching method (Figure S11) and 
compared with the absolute FRET efficiencies measured by fluorescence spectroscopy in test tube. This comparison enables quantitative evaluation of the binding processes, accounting for the variations caused by the degradation of the oligonucleotide scaffold and photophysical effects of the donor and acceptor.

The FRET efficiency was measured in 300 fixed single cells for each concentration at different time points ranging from 0 to $24 \mathrm{~h}$. It is accepted that fixed cells are representative of live cells as they retain the structural features of formed biomolecular complexes, including those made of proteins and DNA. In addition, possible diffusive processes within the analysed optical section during the photobleaching are minimised. To account for the degradation effect, we processed FRET efficiency data after filtering out the values lower than $20 \%$. This is because we have shown in Figures $2 B$ and S5B that FRET efficiency can vary from 55 to $20 \%$ upon binding, whereas the rapid nuclease-mediated degradation of the Nanoswitch NF- $_{\text {. }}$ кв resulted in FRET values below 20\% (Figure S7).

Figure $3 A-C$ shows the progressive decrease in FRET efficiency, from $50 \%$ (extracellular Nanoswitch NF-KB $_{\text {at }}$ t=0 h) to $25-30 \%$, in the first $7 \mathrm{~h}$, followed by a levelling off in values until $24 \mathrm{~h}$. Of note, lipoplex particles adsorbed on the extracellular matrix were also analysed. The analysis provided the initial FRET

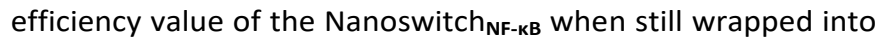
lipofectamine at $t=0$. We measured a FRET value of approximately $50 \pm 10 \%$, which is consistent with the FRET observed in the titration experiments performed by fluorescence spectroscopy (see $\mathrm{C}_{\mathrm{NF}-\mathrm{KB}}=0$ in Figure $2 \mathrm{~B}$ ). This

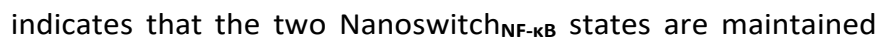
after complexation with lipofectamine and are stable against extracellular nuclease.

Quantitative FRET analysis of the cells treated with the control FRET sequence for 2 and $24 \mathrm{~h}$ (Figure 3D) shows that FRET efficiency (40\%) is constant within the incubation period studied. We could argue that the trend observed in Figure 3A-C is likely due to the conformational change of the Nanoswitch ${ }_{\mathrm{NF}-}$
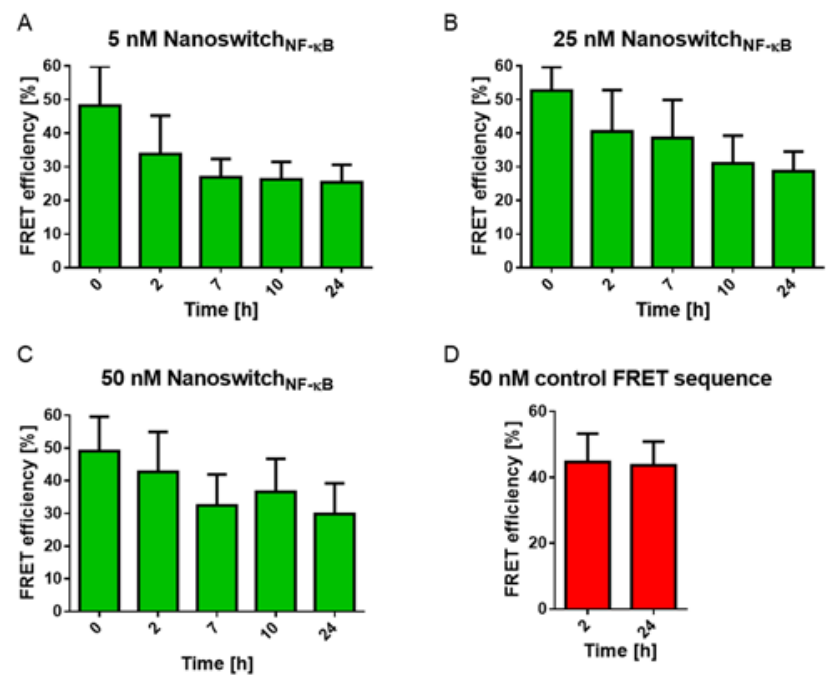

Figure 3. Intracellular FRET measurements in PC3 cells transfected with (A) $5 \mathrm{nM}$, (B) 25 $\mathrm{nM}$ and (C) $50 \mathrm{nM}$ Nanoswitch $_{\mathrm{NF}-\mathrm{kB}}$ or (D) $50 \mathrm{nM}$ control FRET sequence. Error bars represent standard deviations; $\mathrm{n}=80$.

кв induced by binding to $p 50 / p 65$ or p50/p50, which occurred in the first 7-10 h. Importantly, when compared with the data obtained in test tube (Figure 2B), the final FRET efficiency values obtained in cells suggest that the Nanoswitch ${ }_{\text {NF-KB }}$ can adopt the bound conformation. Overall, FRET imaging microscopy revealed that a fraction of Nanoswitch ${ }_{\mathrm{NF}-\mathrm{KB}}$ molecules internalised in the cells are degraded in the endosomes upon intracellular trafficking, while the fraction of molecules released in the cytosol likely binds to the target NF-KB.

We further demonstrated that the intracellular FRET efficiency values are sensitive to the intracellular NF-KB concentration. Cells were transfected with $50 \mathrm{nM}$ siRNAlipofectamine formulation targeting NF-KB and were subsequently transfected with $25 \mathrm{nM}$ Nanoswitch NF-KB $_{\text {(Figure }}$ $\mathrm{S} 12 \mathrm{~A})$. Variation in NF-KB expression induced by the siRNA treatment was probed in live cells by measuring FRET efficiency values. As depicted in Figure S12B, the higher FRET values that were obtained after partial knockdown of the protein were consistent with the lower protein content, which was also confirmed by Western Blot analysis (Figure S12C and D). Of note, transfection of PC3 cells with Nanoswitch ${ }_{\mathrm{NF}-\mathrm{kB}}$ did not affect cell viability or the total intracellular NF-KB protein concentration, as confirmed by the cell viability studies and ELISA (enzymelinked immunosorbent assay) (Figures S13 and S14).

Live cells treated for $2 \mathrm{~h}$ with different concentrations (5-50 $\mathrm{nM}$ ) of Nanoswitch NF-kB $_{\text {B }}$ and control FRET sequence were incubated further for $\mathrm{O} \mathrm{h}$ ( $2 \mathrm{~h}$ timepoint) or $22 \mathrm{~h}$ ( $24 \mathrm{~h}$ timepoint) and imaged (Figure 4) to explore the subcellular localisation of the two species.

As confocal and FRET microscopy analysis suggested that the endosomal escape and binding of Nanoswitch NF-kB $_{\text {occurred }}$ after 2 and $24 \mathrm{~h}$ incubation, respectively, we selected these two time points to compare the behaviour of Nanoswitch NF-KB $_{\text {and }}$ control FRET sequence.

Diffuse cytosolic and nuclear green fluorescence (donor) were noticed (Figures 4 and S15) as well as punctate fluorescence, suggesting the cytosolic release and nuclear diffusion/translocation of the Nanoswitch NF-KB $_{\text {. The punctate }}$ signal may indicate either residual endo-lysosomal confinement of the Nanoswitch NF-kB $_{\text {or }}$ or the presence of cytosolic nanocomplexes composed of Nanoswitch $\mathrm{NF}_{\mathrm{NFB}}$ and the cationic lipids. Approximately $28 \%, 51 \%$ and $76 \%$ of cells transfected with 5, 25 and $50 \mathrm{nM}$ Nanoswitch NF-KB $_{\text {respectively, exhibited }}$ nuclear signal after $2 \mathrm{~h}$ incubation. Prolonged incubation time (24 h) caused a significant drop in number of cells presenting nuclear signal to approximately $0 \%, 2 \%$ and $8 \%$, respectively (Figure S16). Although cells treated with control FRET sequence show punctate and cytosolic dim fluorescence signal, only $6 \%$ of the total analysed cells presented fluorescence in the nucleus after $2 \mathrm{~h}$ transfection and $0.3 \%$ after $24 \mathrm{~h}$ transfection.

Single-stranded and double-stranded oligonucleotides passively diffused and accumulated into the nucleus when directly deployed in the cytosol by microinjection. ${ }^{36}$ Little is known about the diffusive properties of oligonucleotides associated with cationic lipids or proteins. The unassisted diffusion of biomolecules and nanoparticles into the nucleus becomes restricted as they approach a size limit of 


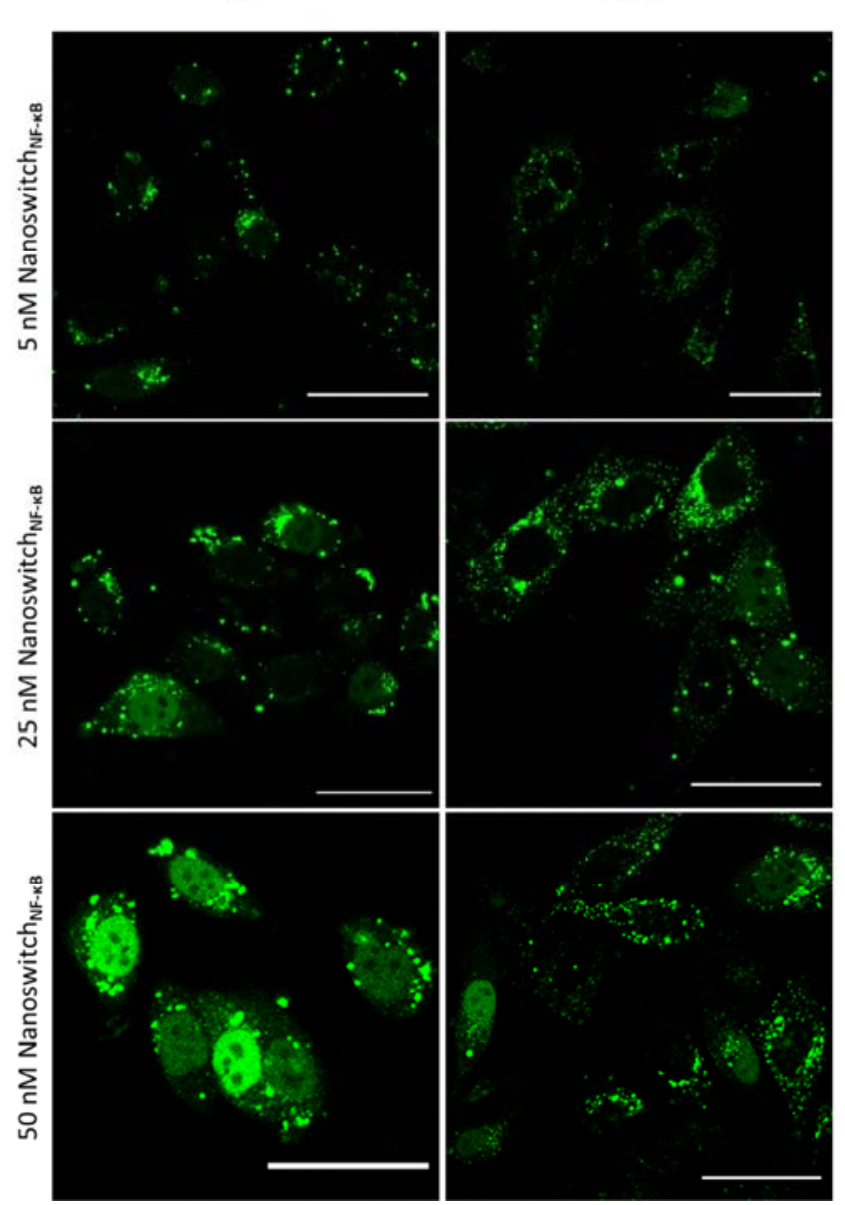

$2 \mathrm{~h}$

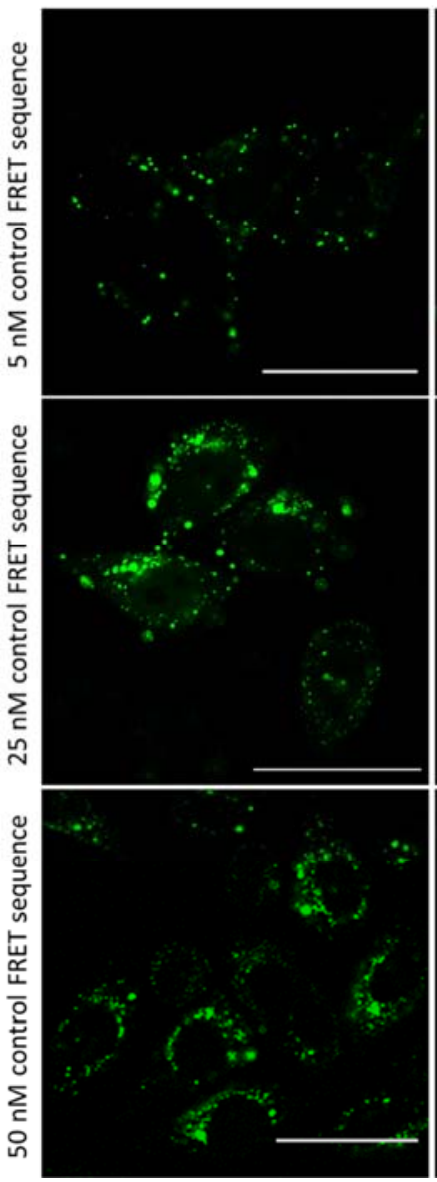

$24 \mathrm{~h}$

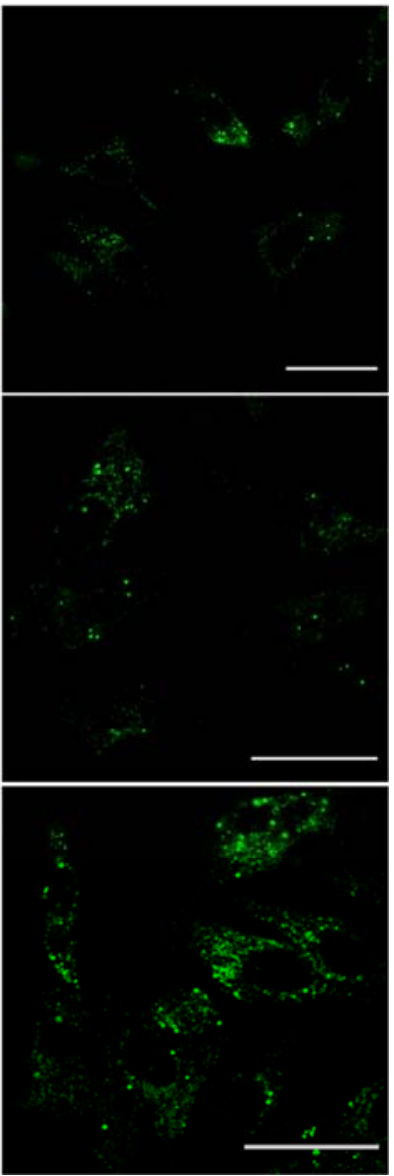

Figure 4. Representative confocal microscopy image of PC3 cells transfected with the $5 \mathrm{nM}, 25 \mathrm{nM}$ and $50 \mathrm{nM}$ Nanoswitch $\mathrm{NF}_{\mathrm{kB}}$ ( $\mathrm{A}$ ) or control FRET sequence (B) live imaged after $2 \mathrm{~h}$ and $24 \mathrm{~h}$. The dim fluorescence signal of the control FRET sequence was acquired using higher laser intensity. Scale bar $=50 \mu \mathrm{m}$.

approximately $10 \mathrm{~nm}$ in diameter. ${ }^{37}$ In contrast, protein complexes such as NF-KB after activation, are ushered selectively by dedicated transport receptors, which recognise specific import signal (NLS) peptides displayed by the cargo. ${ }^{37}$

The observations in live cells suggest that, compared to the

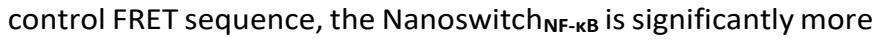
prone to bind to the activated NF-KB in the cytosol and be translocated into the nucleus. However, the nuclear localisation appears to be a transient process occurring after transfection. Indeed, it has been recently reported that the transfection of cells with Lipofectamine and nucleic acids further activate the intracellular NF-KB signalling pathway. ${ }^{38}$ After cellular activation, NF-KB is shuttled to the nucleus, and the binding of the NF-KB to its gene targets generally results in the transcription and synthesis of $1 \kappa B$ protein. This protein promptly recognises the NF-KB dimers and re-shuttles them back to the cytosol. ${ }^{39,40}$ This may explain the observed transient localisation of fluorescent signal in the nuclei and the disappearance of the signal may indicate that the Nanoswitch ${ }_{\mathrm{NF}-\mathrm{kB}} / \mathrm{NF}-\mathrm{KB}$ complex can be actively relocated in the cytosol.

Super-resolution visualisation of the interactions between NF-kB and Nanoswitch ${ }_{\mathrm{NF}-\mathrm{KB}}$
To directly visualise the specific binding of Nanoswitch $\mathrm{NF}_{\mathrm{K}-\mathrm{K}}$ to NF-кB at the molecular level and with nanometer resolution, we employed stochastic optical reconstruction microscopy ( $\mathrm{N}$ STORM). This super-resolution microscopy technique allows for simultaneous multicolour imaging of subcellular nanostructures and nanomaterials with approximately $20 \mathrm{~nm}$ lateral resolution, due to the accurate localisation of randomly blinking fluorescent molecules. ${ }^{41-43}$

The Nanoswitch ${ }_{\text {NF-KB }}$ was designed with the activatorreporter pair (Quasar 570- Quasar 670), which is suitable for $\mathrm{N}$ STORM multi-channel acquisition. We used 2D STORM-TIRF microscopy to maximise signal-to-noise ratio and simultaneously image Nanoswitch ${ }_{\mathrm{NF}-\mathrm{KB}}$ and NF-KB molecules in approximately $200 \mathrm{~nm}$ layer in the cytosol. Panels A-D in Figure 5 show representative STORM images of PC3 cell transfected for $2 \mathrm{~h}$ with $50 \mathrm{nM}$ Nanoswitch ${ }_{\mathrm{NF}-\mathrm{KB}}$ and control FRET sequence, followed by additional $2 \mathrm{~h}$ and $22 \mathrm{~h}$ incubation in fresh culture medium.

At both early and late stage of incubation, STORM images revealed widespread Nanoswitch NF-kB $_{\text {molecules as well as }}$ individual red nanometric spots, indicating the presence of Nanoswitch $_{\text {NF-KB }}$ clusters (red signal Figure 5A, B and Figure 6A) approximately $90 \mathrm{~nm} \pm 60 \mathrm{~nm}$ in size. These nanoclusters can be likely ascribed to the lipofectamine-Nanoswitch ${ }_{\text {NF-KB }}$ complexes, 


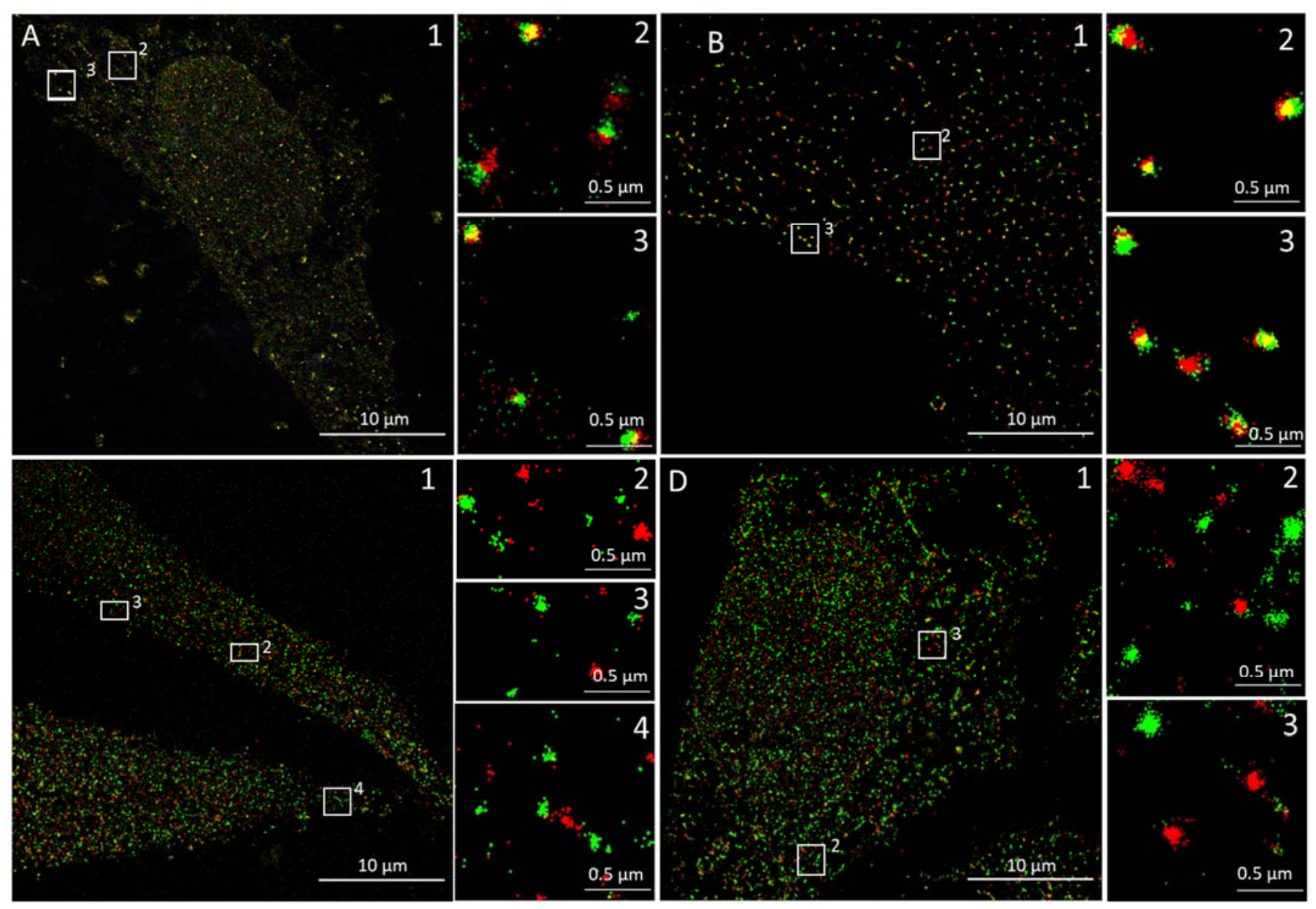

Figure 5. Multicolour STORM super-resolution images of representative PC3 cells transfected with $50 \mathrm{nM}$ Nanoswitch $\mathrm{NF}_{\mathrm{NB}}$ for (A) $4 \mathrm{~h}$ or (B) $24 \mathrm{~h}$ and $50 \mathrm{nM}$ control FRET sequence for (C) $4 \mathrm{~h}$ or (D) $24 \mathrm{~h}$. (1) Large view of a representative PC3 cell. Image 2-4: high magnification images showing details of identified objects. The green signal denotes NF-KB p50

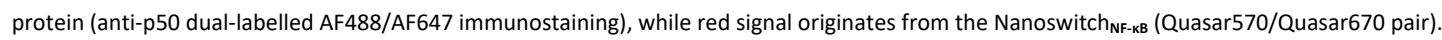

incorporating multiple Nanoswitch ${ }_{\mathrm{NF}-\mathrm{KB}}$ copies, that are imaged when still confined in the endo-lysosome vesicles or after escaping into the cytosol. The dissociation of DNA from cationic lipids is a crucial step for delivery of Nanoswitch ${ }_{\mathrm{NF}-\mathrm{kB}}$, as only the freed molecules can potentially interact with the cytosolic NFkB. ${ }^{44}$

The imaging of NF-KB p50 dimers was performed using a primary polyclonal anti-NF-KB antibody, combined with an AF488/AF647 dual-labelled secondary antibody. The STORM imaging of NF-KB molecules resulted in nanoobjects approximately $80 \mathrm{~nm} \pm 50 \mathrm{~nm}$ in size (green signal Figures 5 and $6)$. By counting the localizations in the region of interest for the majority of nanoobjects, we identified $60 \pm 30$ molecules within an imaged nanoobject (Figure S17). This suggests the presence of higher-order aggregates of NF-KB molecules. It is worth noting that the Nanoswitch ${ }_{\mathrm{NF}-\mathrm{kB}}$ and anti-NF-KB polyclonal antibody have different docking sites on the protein. While the anti-p50 polyclonal antibody targets an internal region of the NF-KB p50 protein, the DNA binds to the N-terminal domains of the NF-KB dimers. ${ }^{45}$

STORM images of PC3 cells incubated for $2 \mathrm{~h}$ and $24 \mathrm{~h}$ with Nanoswitch NF-KB $_{\text {B }}$ also revealed the presence of numerous multicolour red/yellow/green nanometric assemblies (Figure 5A, B and $6 \mathrm{C}$ ) indicating colocalisation between NF-KB clusters and

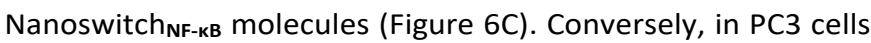
incubated with the control FRET sequence for 2 and $24 \mathrm{~h}$, a large amount of green signal was not associated with the NF-KB nanocluster and single molecules (Figures $5 C, D$ and $6 C$ ). Figure 6C shows that after $2 \mathrm{~h}$ incubation the colocalisation between Nanoswitch $\mathrm{NF}_{\mathrm{NFB}}$ and NF-KB molecules is approximately $60 \%$ and does not change significantly at prolonged incubation times. The colocalisation of the control FRET sequence with NF-KB was limited to $25-30 \%$. This limited colocalisation might result from non-specific weak binding of NF-kB to the control sequence, driven by electrostatic interactions, in agreement with the results obtained in test tube.

Overall STORM based super-resolution imaging enabled the direct visualisation of the specific molecular interactions between Nanoswitch NF-kB $_{\text {B }}$ and transcription factor proteins in the cytosol with nanometric resolution. This supported our FRET microscopy study confirming the hypothesis that the Nanoswitch $_{\text {NF-kB }}$ molecules deployed in the cytosol are able to target NF-KB.

\section{Visualisation of intracellular bound and unbound Nanoswitch $\mathrm{N}_{\mathrm{NF}-\mathrm{KB}}$ molecules by FLIM}



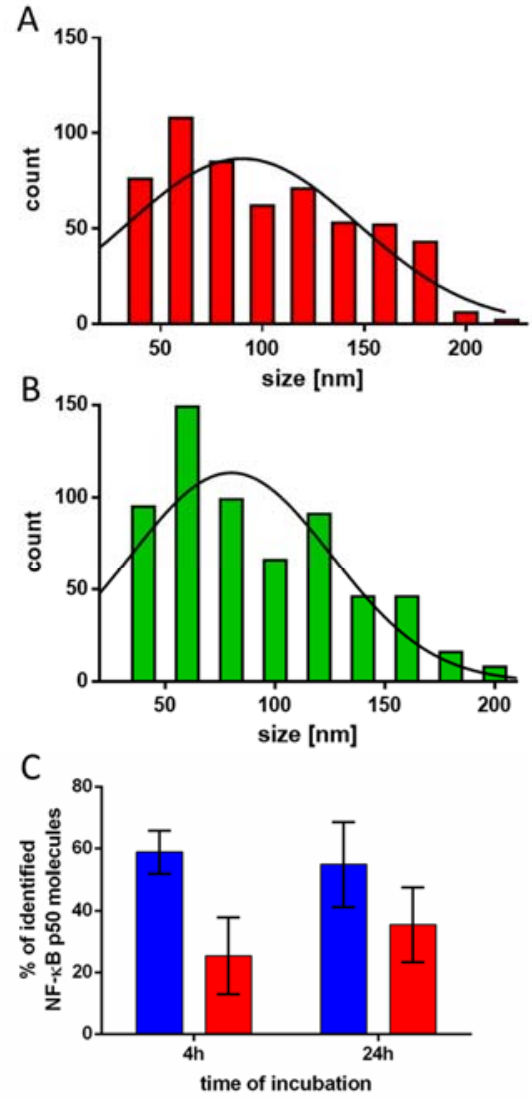

- Nanoswitch ${ }_{\mathrm{NF} \times \mathrm{B}}=$ FRET negative control
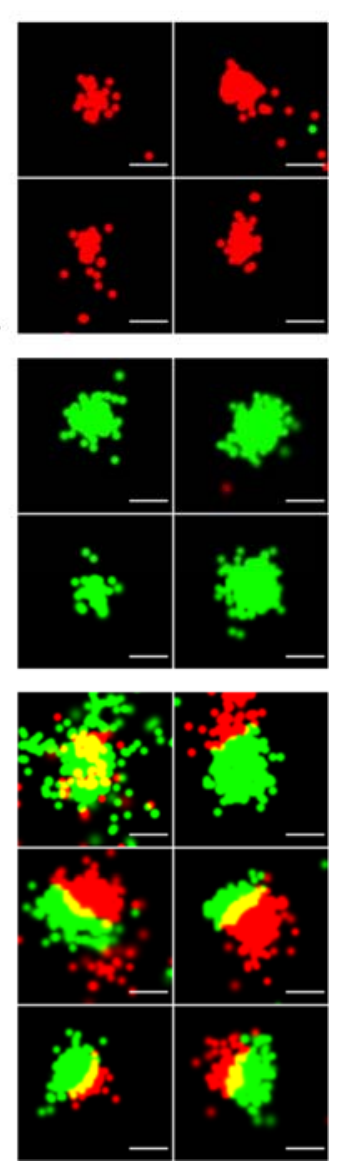

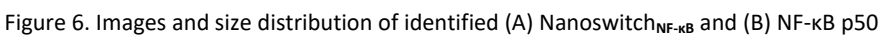
molecules; $n=110$. (C) Statistical analysis of STORM images describing the extent of

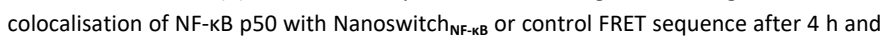
$24 \mathrm{~h}$ transfection. Error bars denote standard deviation; $\mathrm{n}=5$. Images denote examples of colocalised nano-assemblies. Scale bar $=100 \mathrm{~nm}$.
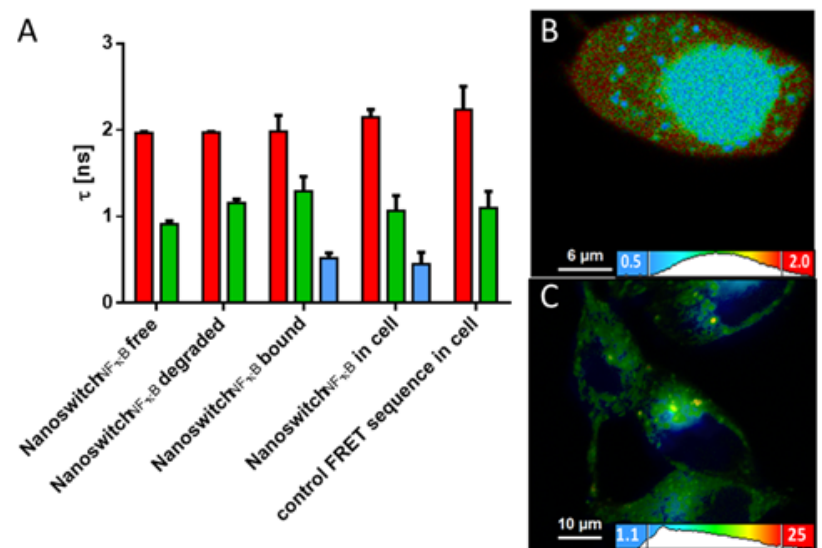

Figure 7. (A) Fluorescence lifetime, $\tau$, of solutions of free $5 \mathrm{nM} \mathrm{Nanoswitch}$ NF-kB (Nanoswitch $_{\text {NF-KB }}$ free), $5 \mathrm{nM}$ Nanoswitch ${ }_{\text {NF-KB }}$ degraded by DNase I (Nanoswitch NF-KB $_{\text {.KB }}$

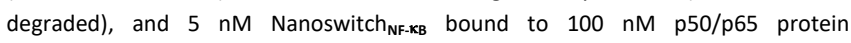
(Nanoswitch $_{\mathrm{NF}-\mathrm{KB}}$ bound), and $\tau$ measured in cells transfected with $50 \mathrm{nM}$ Nanoswitch $_{\mathrm{NF}-\mathrm{KB}}$ (Nanoswitch $\mathrm{NF-KB}_{\text {B }}$ in cell) or $50 \mathrm{nM}$ control FRET sequence (control FRET sequence in cell). Error bars represent standard deviations; $n=20$. (B) Representative FLIM image acquired in live PC3 cells incubated with $50 \mathrm{nM}$ Nanoswitch $_{\mathrm{NF}-\mathrm{kB}}$ for $2 \mathrm{~h}$. (C) Representative FLIM image acquired in live PC3 cells incubated with $50 \mathrm{nM}$ control FRET sequence for $2 \mathrm{~h}$.
Intracellular FLIM measurements were performed to correlate the sub-cellular distribution of Nanoswitch $\mathrm{NF}_{\mathrm{NB}}$ and its binding activity in living cells. As the donor time decay either in the binding competent state or bound state (see equilibrium in Figure 1) is significantly affected by FRET quenching mechanism (FRET $20 \%$ ), we probed the time decay of the acceptor, which is not influenced by the energy transfer process and can be easily used as a parameter to independently probe the binding of Nanoswitch NF-KB $_{\text {to }}$ the protein.

The fluorescence time decays of the untreated and DNase treated Nanoswitch ${ }_{\mathrm{NF}-\mathrm{KB}}$ were first analysed in solution. As shown in Figure 7A, both samples featured a bi-exponential decay profile when Quasar 670 was excited with a pulsed 640 $\mathrm{nm}$ laser. The intact and degraded Nanoswitch $\mathrm{NF-KB}_{\mathrm{K}}$ showed two fluorescence lifetimes i.e., $0.91 \pm 0.04 \mathrm{~ns}$ and $1.97 \pm 0.02 \mathrm{~ns}$, and $1.16 \pm 0.04$ ns and $1.97 \pm 0.01 \mathrm{~ns}$, respectively. Hence, the two lifetimes can be ascribed to the intrinsic photophysical and conformational properties of the modified Quasar670.

After addition of $100 \mathrm{nM}$ protein to the solution of $5 \mathrm{nM}$ Nanoswitch $_{\mathrm{NF}-\mathrm{kB}}$, the fluorescence changed to a tri-exponential decay profile, with lifetimes of $2.0 \pm 0.2 \mathrm{~ns}, 1.3 \pm 0.2 \mathrm{~ns}$, and 0.52 $\pm 0.06 \mathrm{~ns}$. In addition to the two lifetimes already observed for the free Nanoswitch ${ }_{\text {NF-kB }}$, a short-lived component was observed. We ascribed the longer lifetimes to the unbound Nanoswitch $_{\text {NF-kB }}$ molecules and the shortest lifetime to the

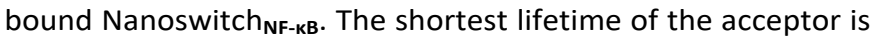
related to quenching processes typically mediated by a variety of molecular interactions and rearrangements taking place upon binding to the protein.

A tri-exponential decay fluorescence profile was also observed in the cells transfected with $50 \mathrm{nM}$ Nanoswitch ${ }_{\mathrm{NF}-\mathrm{KB} \text {, }}$ as shown in the FLIM image (Figure 7B). Three lifetimes, $2.1 \pm$ $0.3 \mathrm{~ns}$ (red), $1.1 \pm 0.2 \mathrm{~ns}$ (green), and $0.4 \pm 0.2 \mathrm{~ns}$ (light blue) (Figure S18) were measured in the cells and the relative abundances of the three observed decay times were $40 \%, 40 \%$ and $20 \%$, respectively.

The intracellular lifetime values are consistent with the FLIM results obtained in solution, indicating the presence of both protein-unbound and protein-bound Nanoswitch NF-KB. $_{\text {. }}$ Interestingly, FLIM imaging revealed that the Nanoswitch NF-KB localised in the nucleus binds to the target protein. To confirm this, FLIM experiments were carried out on cells transfected with $50 \mathrm{nM}$ control non-binding FRET sequence. FLIM images indicated lack of signal in the nucleus and a bi-exponential decay profile for the acceptor with lifetimes of ca. $1.0 \pm 0.1$ and $2.1 \pm 0.1 \mathrm{~ns}$ (Figure 7C and S18) as expected in the absence of binding interactions. Overall, these results are consistent with the previous finding and highlight the use of FLIM microscopy for real-time probing of the binding processes and subcellular distribution of Nanoswitch $\mathrm{NF-KB}_{\text {B }}$ in single living cells.

\section{Tracking Nanoswitch $\mathrm{NF}_{\mathrm{NB}}$-protein interactions and degradation in live cells by FCS}

Fluorescence Correlation Spectroscopy (FCS), an extremely sensitive and non-invasive technique with high spatio-temporal resolution, ${ }^{46,47}$ was used to discriminate between three species 
i.e., unbound, bound, and degraded Nanoswitch ${ }_{\mathrm{NF}-\mathrm{KB}}$ in different cellular compartments based on their different diffusional properties (Table 1).

First, the diffusion coefficient (D) of the Nanoswitch ${ }_{\text {NF-KB }}$ and control FRET sequence were measured in solution. To extrapolate the diffusion coefficients from the acquired correlation functions, the confocal volume $(0.7 \mathrm{fL})$ was determined using AF555 dye as a reference probe. The autocorrelation curves were best fitted with a Brownian diffusion model including triplet dynamic and two components. Table 1 shows the measured diffusion coefficients for the Nanoswitch $_{\text {NF-KB }}$ in the unbound, complexed, and degraded states. The Nanoswitch NF-kB $_{\text {in }}$ in solution showed a $D$ of $60 \pm$ $20 \mu \mathrm{m}^{2} / \mathrm{s}$. This is in agreement with data reported in previous studies on size-dependent DNA mobility. ${ }^{36}$ However, a considerably lower $D$ of $35 \pm 7 \mu \mathrm{m}^{2} / \mathrm{s}$ was measured for the

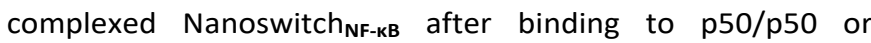

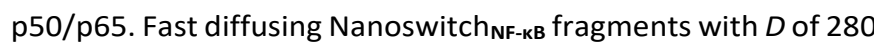
$\pm 100 \mu \mathrm{m}^{2} / \mathrm{s}$ were observed after DNAse treatment. We found that the diffusivities of the free and degraded control FRET sequence (Table 1) were comparable to the ones observed for the Nanoswitch ${ }_{\mathrm{NF}-\mathrm{kB}}$, with values of $70 \pm 20 \mu \mathrm{m}^{2} / \mathrm{s}$ and $290 \pm 170$ $\mu \mathrm{m}^{2} / \mathrm{s}$, respectively. Conversely, after incubation with the p50/p50 protein, the diffusion coefficient of control FRET sequence remained unchanged. This is due to the limited binding of the control FRET sequence to the targeting protein.

Next, the diffusion coefficients and relative abundance of each species were measured in live cells after $4 \mathrm{~h}$ and $24 \mathrm{~h}$ incubation. Transfection with $50 \mathrm{nM}$ Nanoswitch NF-kB $_{\text {or control }}$ FRET sequence was performed for $2 \mathrm{~h}$, then the medium was discarded, cells were cultured for further $2 \mathrm{~h}$ and $22 \mathrm{~h}$ in fresh culture medium and analysed by FCS. Experimental autocorrelation curves (Figure S19) were best fitted with an anomalous diffusion model including the triplet dynamic and two components, where the dye is diffusing in three dimensions through a crowded cellular environment. ${ }^{48}$ Bi-exponential autocorrelation curves were systematically observed in live cells, after $4 \mathrm{~h}$ incubation, with two diffusing species, ascribed to either bound and free Nanoswitch NF-KB $\left(D=3 \mu \mathrm{m}^{2} / \mathrm{s}\right.$ and 40 $\mu \mathrm{m}^{2} / \mathrm{s}$, respectively) or the bound and degraded Nanoswitch $\mathrm{NF}_{\mathrm{N}-}$ кв $\left(D=3 \mu \mathrm{m}^{2} / \mathrm{s}\right.$ and $\left.320 \mu \mathrm{m}^{2} / \mathrm{s}\right)$ (Table 1$)$. We observed similar $D$ values at prolonged incubation times. The most abundant species observed in the cells was the slow diffusing species with a fractional abundance ranging between 60 and $100 \%$. However, it is worth mentioning that contribution of a given population of fluorescent species to the autocorrelation function depends on both the concentration and brightness. ${ }^{49}$ As shown in Table 1, we did not observe a significant difference in the intracellular diffusional properties of Nanoswitch NF-kB $_{\text {B }}$ and control FRET sequence. We infer that by FCS we can mainly probe the diffusivity $\left(3 \mu \mathrm{m}^{2} / \mathrm{s}\right)$ of the intracellular DNAlipofectamine nanocomplexes that we also captured by STORM imaging. These nanocomplexes are likely localised in the cytosol rather than confined into the endo-lysosomes. In fact, the intracellular mobility of the lysosomal vesicles is typically two orders of magnitude lower $\left(0.071 \mu \mathrm{m}^{2} / \mathrm{s}\right)^{50}$ and we did not
Table 1. Fluorescence correlation spectroscopy measurements of diffusion coefficients

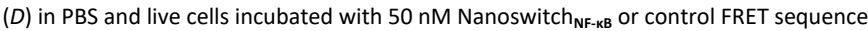
for $4 \mathrm{~h}$.

\begin{tabular}{ccccc}
\hline \multirow{2}{*}{$\boldsymbol{D}\left[\mathrm{\mu m}^{2} / \mathrm{s}\right]$} & \multicolumn{2}{c}{ Nanoswitch } & & \multicolumn{2}{c}{ Control FRET sequence } \\
\cline { 2 - 5 } & PBS & Cell & PBS & Cell \\
\hline Bound DNA & $35 \pm 7$ & $3 \pm 2$ & $60 \pm 20$ & $4 \pm 2$ \\
\hline Free DNA & $60 \pm 20$ & $40 \pm 30$ & $70 \pm 20$ & $40 \pm 20$ \\
\hline DNA degraded & $280 \pm 100$ & $320 \pm 230$ & $290 \pm 170$ & $220 \pm 120$ \\
\hline
\end{tabular}

observe such a component in the correlation curve. This is in agreement with our previous studies, where we determined that the limiting step for nucleic acid intracellular delivery is its disassociation from the carrier in the cytosol rather than endosomal escape. ${ }^{44}$

We found that the freely diffusing species $\left(40 \mu \mathrm{m}^{2} / \mathrm{s}\right)$, i.e

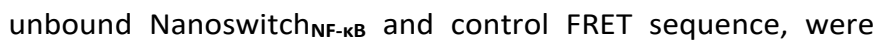
slightly affected by the crowded intracellular environment. The measured $D$ values are consistent with data reported in previous studies on size-dependent DNA mobility, ${ }^{36}$ where it was found that the diffusion of small oligonucleotides in the cytoplasm was mildly impeded and that it was similar to the one measured in water. Finally, the intracellular fast diffusing species (190-270 $\mu^{2} / \mathrm{s}$ ) can be likely assigned to the degraded Nanoswitch $_{\text {NF-кB }}$ or control FRET sequence. Overall, the FCS studies in live cells provided an insight into the mobility of the cytosolic and degraded fractions of Nanoswitch NF-KB $_{\text {and }}$ confirmed the presence of oligonucleotides molecules stably bound to the cationic lipids after the escaping from the endosomes. Unlike the FRET microscopy study, which enabled the discrimination of the Nanoswitch ${ }_{\text {NF-kB }}$ molecules bound to

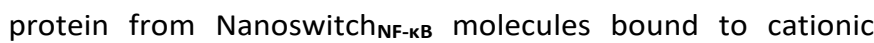
lipids, FCS analysis does not allow us to distinguish between similar slow diffusing complexes.

\section{Conclusions}

The present study highlights the importance of combining quantitative and super-resolution microscopy approaches for monitoring the intracellular behaviour and signalling efficiency of DNA nanosensors. The use of multiple microscopy techniques is a powerful strategy to evaluate non-specific and false-positive signals resulting from degradation or off-target effects. We have shown by quantitative FRET microscopy that functional Nanoswitch $_{\text {NF-KB }}$ molecules delivered in the cytosol effectively bind to NF-KB proteins present in the cytosol and in the nucleus. By employing super-resolution imaging of cells, we demonstrated the nanoscale colocalisation of the DNA nanosensors and its target protein, providing direct evidence of their molecular interaction. Furthermore, by STORM microscopy and FCS we found that after escape from the endolysosomal vesicles, the DNA nanosensor remained entangled with the transfection agent into nanoclusters. This combined approach can be extended to other DNA-nanostructures to dissect their intracellular spatial and spatiotemporal distribution and functionality, allowing for evaluation of the sensing properties of a multitude of DNA-based devices. 


\section{Conflicts of interest}

There are no conflicts to declare.

\section{Acknowledgements}

This work was funded under an Australian Research Council (ARC) Future Fellowship scheme (F. Cavalieri FT140100873) and an Establishment Grant by The University of Melbourne ( $F$. Cavalieri, A. Glab). F. Caruso acknowledges the award of a National Health and Medical Research Council Senior Principal Research Fellowship (GNT1135806). This project received funding from the European Union's Horizon 2020 research and innovation programme under the Marie Skłodowska-Curie grant agreement No. 690901 (NANOSUPREMI). The research was also partly funded by the ARC Centre of Excellence in Convergent Bio-Nano Science and Technology (Project No. CE140100036). This work was performed in part at the Materials Characterisation and Fabrication Platform (MCFP) at The University of Melbourne. A. Bertucci acknowledges funding from the European Union's Horizon 2020 research and innovation program under the Marie Skłodowska-Curie grant agreement No. 704120 ("MIRNANO"). A. Amodio acknowledges funding from the European Union's Horizon 2020 research and innovation program under the Marie Skłodowska-Curie grant agreement No. 798565 ("RE-IMMUNE"). We acknowledge Marco Savioli for providing help with the fluorescence measurements.

\section{Notes and references}

1. Q. Hu, H. Li, L. Wang, H. Gu and C. Fan, Chem Rev, 2019, 119, 6459-6506.

2. N. C. Seeman and H. F. Sleiman, Nat Rev Mater, 2017, 3, 17068.

3. Y. J. Chen, B. Groves, R. A. Muscat and G. Seelig, Nat Nanotechnol, 2015, 10, 748-760.

4. M. Kahan-Hanum, Y. Douek, R. Adar and E. Shapiro, Sci Rep-Uk, 2013, 3, 1535.

5. J. D. Munzar, A. Ng and D. Juncker, Chem Soc Rev, 2019, 48, 1390-1419.

6. S. Modi, C. Nizak, S. Surana, S. Halder and Y. Krishnan, Nat Nanotechnol, 2013, 8, 459-467.

7. A. Bertucci, J. Guo, N. Oppmann, A. Glab, F. Ricci, F. Caruso and F. Cavalieri, Nanoscale, 2018, 10, 2034-2044.

8. F. Wang, X. Liu and I. Willner, Angew Chem Int Ed Engl, 2015, 54, 1098-1129.

9. A. Vallee-Belisle, A. J. Bonham, N. O. Reich, F. Ricci and K. W. Plaxco, J Am Chem Soc, 2011, 133, 13836-13839.

10. H. Asanuma, K. Murayama, Y. Kamiya and H. Kashida, $B$ Chem Soc Jpn, 2018, 91, 1739-1748.

11. H. Pei, L. Liang, G. Yao, J. Li, Q. Huang and C. Fan, Angew Chem Int Ed Engl, 2012, 51, 9020-9024.

12. N. Narayanaswamy, K. Chakraborty, A. Saminathan, E. Zeichner, K. Leung, J. Devany and Y. Krishnan, Nat Methods, 2019, 16, 95-102.

13. K. Leung, K. Chakraborty, A. Saminathan and Y. Krishnan, Nat Nanotechnol, 2019, 14, 176-183.
14.

M. You, Y. Lyu, D. Han, L. Qiu, Q. Liu, T. Chen, C. Sam Wu, L. Peng, L. Zhang, G. Bao and W. Tan, Nat Nanotechnol, 2017, 12, 453-459.

15. L. Nuhn, S. Van Herck, A. Best, K. Deswarte, M. Kokkinopoulou, I. Lieberwirth, K. Koynov, B. N. Lambrecht and B. G. De Geest, Angew Chem Int Ed Engl, 2018, 57, 10760-10764.

16. Y. Ma, Y. Yamamoto, P. R. Nicovich, J. Goyette, J. Rossy, J. J. Gooding and K. Gaus, Nat Biotechnol, 2017, 35, 363-370.

17. C. E. Rowland, C. W. Brown, I. L. Medintz and J. B. Delehanty, Methods Appl Fluoresc, 2015, 3, 042006.

18. M. Czarnek and J. Bereta, Sci Rep-Uk, 2017, 7, 11682.

19. D. Mason, G. Carolan, M. Held, J. Comenge, S. Cowman and R. Lévy, ScienceOpen Research, 2016, 0, 1-10.

20. X. A. Wu, C. H. Choi, C. Zhang, L. Hao and C. A. Mirkin, J Am Chem Soc, 2014, 136, 7726-7733.

21. M. Hirsch and M. Helm, Nucleic Acids Res, 2015, 43, 46504660.

22. A. Sasaki and M. Kinjo, J Control Release, 2010, 143, 104111.

23. S. Tyagi, Nat Methods, 2009, 6, 331-338.

24. A. Lacroix, E. Vengut-Climent, D. de Rochambeau and H. F. Sleiman, ACS Cent Sci, 2019, 5, 882-891.

25. G. Ghosh, V. Y. Wang, D. B. Huang and A. Fusco, Immunol Rev, 2012, 246, 36-58.

26. M. Karin, Y. Cao, F. R. Greten and Z. W. Li, Nat Rev Cancer, 2002, 2, 301-310.

27. F. E. Chen, D. B. Huang, Y. Q. Chen and G. Ghosh, Nature, 1998, 391, 410-413.

28. Z. Q. W. Zhao, R. Roy, J. C. M. Gebhardt, D. M. Suter, A. R. Chapman and X. S. Xie, Proceedings of the National Academy of Sciences of the United States of America, 2014, 111, 681-686.

29. D. B. Huang, C. B. Phelps, A. J. Fusco and G. Ghosh, J Mol Biol, 2005, 346, 147-160.

30. S. A. Marras, F. R. Kramer and S. Tyagi, Nucleic Acids Res, 2002, 30, e122.

31. A. Pomorski, T. Kochanczyk, A. Miloch and A. Krezel, Anal Chem, 2013, 85, 11479-11486.

32. I. M. Kuznetsova, K. K. Turoverov and V. N. Uversky, Int J Mol Sci, 2014, 15, 23090-23140.

33. S. Nakano, D. Miyoshi and N. Sugimoto, Chem Rev, 2014 114, 2733-2758.

34. D. M. Presman, D. A. Ball, V. Paakinaho, J. B. Grimm, L. D. Lavis, T. S. Karpova and G. L. Hager, Methods, 2017, 123, 76-88.

35. D. Mazza, A. Abernathy, N. Golob, T. Morisaki and J. G. McNally, Nucleic Acids Res, 2012, 40, e119.

36. G. L. Lukacs, P. Haggie, O. Seksek, D. Lechardeur, N. Freedman and A. S. Verkman, J Biol Chem, 2000, 275, 16251629.

37. B. Naim, V. Brumfeld, R. Kapon, V. Kiss, R. Nevo and Z. Reich, J Biol Chem, 2007, 282, 3881-3888.

38. X. Guo, H. Wang, Y. Li, X. Leng, W. Huang, Y. Ma, T. Xu and X. Qi, Immunol Cell Biol, 2019, 97, 92-96.

39. R. E. Lee, S. R. Walker, K. Savery, D. A. Frank and S. Gaudet, Mol Cell, 2014, 53, 867-879.

40. M. G. Dorrington and I. D. C. Fraser, Front Immunol, 2019, 10, 705 .

M. Bates, B. Huang, G. T. Dempsey and X. Zhuang, Science, 2007, 317, 1749-1753. 
42. M. J. Rust, M. Bates and X. Zhuang, Nat Methods, 2006, 3, 793-795.

43. X. Zhuang, Nat Photonics, 2009, 3, 365-367.

44. M. Wojnilowicz, A. Glab, A. Bertucci, F. Caruso and F. Cavalieri, ACS Nano, 2019, 13, 187-202.

45. D. J. Hart, R. E. Speight, M. A. Cooper, J. D. Sutherland and J. M. Blackburn, Nucleic Acids Res, 1999, 27, 1063-1069.

46. N. Altan-Bonnet and G. Altan-Bonnet, Curr Protoc Cell Biol, 2009, Chapter 4, Unit 424.

47. S. A. Kim, K. G. Heinze and P. Schwille, Nat Methods, 2007, 4, 963-973.

48. M. Wachsmuth, W. Waldeck and J. Langowski, J Mol Biol, 2000, 298, 677-689.

49. X. Zhang, A. Poniewierski, K. Sozanski, Y. Zhou, A. Brzozowska-Elliott and R. Holyst, Phys Chem Chem Phys, 2019, 21, 1572-1577.

50. D. Bandyopadhyay, A. Cyphersmith, J. A. Zapata, Y. J. Kim and C. K. Payne, PLoS One, 2014, 9, e86847. 


\section{University Library}

\section{- M M N E R VA A gateway to Melbourne's research publications}

Minerva Access is the Institutional Repository of The University of Melbourne

\section{Author/s:}

Glab, A;Bertucci, A;Martino, F;Wojnilowicz, M;Amodio, A;Venanzi, M;Ricci, F;Forte, G;Caruso, F;Cavalieri, F

Title:

Dissecting the intracellular signalling and fate of a DNA nanosensor by super-resolution and quantitative microscopy.

Date:

2020-07-13

Citation:

Glab, A., Bertucci, A., Martino, F., Wojnilowicz, M., Amodio, A., Venanzi, M., Ricci, F., Forte, G., Caruso, F. \& Cavalieri, F. (2020). Dissecting the intracellular signalling and fate of a DNA nanosensor by super-resolution and quantitative microscopy.. Nanoscale, 12 (28), pp.15402-15413. https://doi.org/10.1039/dOnr03087b.

Persistent Link:

http://hdl.handle.net/11343/241571 